\title{
Statistical Reliability Analysis of Interplanetary Spacecraft Operating at Different Interplanetary Extremity
}

\author{
Malaya Kumar Biswal $\mathbf{M}^{*}$ \\ Department of Physics, \\ School of Physical Chemical and Applied Sciences, \\ Pondicherry University, Kalapet, Puducherry - 605 014, India.
}

\begin{abstract}
Reliability of the spacecraft determines the extent of success probability and mission accomplishment. Despite effective testing and integration, the complexity of the space environment affects reliability. In this paper, we investigate the reliability behaviour of interplanetary spacecraft operating at different interplanetary extremities. So, our investigation assesses spacecraft inhered in interplanetary space with the context of the interplanetary boundary (between distinct planetary orbit or within the bounds of heliopause). From the perspective of spacecraft reliability in interplanetary space, we have excluded planetary landers, atmospheric probes, and satellites maneuvering earth orbit. Thus, we have identified 131 spacecraft (includes 82 probes within the bounds of Sun and the Earth, and 49 within the bounds of Earth and Heliopause) along with their gross mass at launch and lifespan. Based on acquired data, we first conduct a non-parametric analysis of spacecraft reliability to obtain two reliability curves for distinct interplanetary extremity. We then perform a parametric fit (Weibull Distribution) over the data to show the analogy of reliability behaviour. Results showed that the spacecraft operating beyond the extremity of the Earth and the Mars exhibits increased reliability than any other interplanetary extremity. In addition to this, we execute reliability analysis over spacecraft of various mass categories (Small-Medium-Large) to testify the reliability effect interpreted by Dubos in 2010. Finally, we discuss the possible factors and causes accountable for the difference in reliability behaviour concerning the spacecraft design and integration, testing, and constraints in considering spacecraft mass.
\end{abstract}

\section{Nomenclature}

$\begin{array}{ll}\overline{\mathrm{R}} & =\text { Mean Reliability of the Spacecraft } \\ \widehat{\mathrm{R}}(\mathrm{t}) & =\text { Reliability function (Kaplan-Meier) } \\ \mathrm{S}_{\mathrm{f}}(\mathrm{t}) & =\text { Weibull Distribution function for the spacecraft } \\ \mathrm{EH} & =\text { Earth-Heliopause } \\ \mathrm{LCL} & =\text { Lower Confidence Level } \\ \mathrm{R}(\mathrm{t}) & =\text { Weibull Reliability function } \\ \mathrm{SE} & =\text { Sun-Earth } \\ \mathrm{t} & =\text { Lifespan of the Spacecraft (Years) } \\ A \mathrm{U} & =\text { Astronomical Unit }\end{array}$

$$
\begin{array}{ll}
\text { Var } & =\text { Variance } \\
\Gamma & =\text { Gamma function } \\
\lambda(\mathrm{t}) & =\text { Hazard function / Hazard rate } \\
\beta & =\text { Shape parameter } \\
\mathrm{UCL} & =\text { Upper Confidence Level } \\
\eta & =\text { Scale parameter } \\
\sigma & =\text { Standard Deviation } \\
\tau & =\text { Mean life of the spacecraft } \\
\text { ESA } & =\text { European Space Agency }
\end{array}
$$

A. Interplanetary Boundary Condition

- SE-Extremity: The region of interplanetary space extending from the proximity of the Sun to the Earth.

- EH-Extremity: The region of interplanetary space extending from the proximity of the Earth to the farthest point of the Solar System say Heliopause.

\footnotetext{
* Master of Science Student, Department of Physics, Pondicherry University, Kalapet, Puducherry, India. Student Member AIAA (Membership No: 908157). Email: malaykumar1997@gmail.com; mkumar97.res@ pondiuni.edu.in
} 


\section{Introduction}

Reliability of the spacecraft is the key parameter to determine the extent of mission accomplishment and any disruption in reliability may result in spacecraft failures. Scientists and Data analysts have limited their reliability analysis to earthbound satellites [1-6] and their studies to on-orbit spacecraft failures [3]. From the perspective of spacecraft reliability operating at different interplanetary boundary and space environments, no statistical analysis has been found in any of the past technical literature. So, to sustain the stride of reliability analysis and to assert the reliability effect established by Dubos in 2010, we have collected the spacecraft data (in terms of gross mass at launch and lifespan in years) that were launched between 1960 to 2020 from [7-9] and appropriate online resources of space agencies to perform the non-parametric and parametric estimation. Our analysis showed that the spacecraft operating beyond the extremity of the Earth and Mars exhibits maximum reliability as compared to any other interplanetary extremity. Finally, we represent the graphical results and discuss the potential causes responsible for the substantial increase in the reliability behaviour of the spacecraft at EH-Extremity and reduced reliability at SE-Extremity.

\section{Research Methodology and Data Description}

\section{A. Research Methodology}

- Our analysis uses spacecraft data of success, partial success, failed (lifespan estimated until its last active state), and ongoing active spacecraft (lifespan estimated based on the elapsed time as of October 2020) inhered in interplanetary space. The data were gathered in terms of spacecraft's gross mass in kilograms and lifespan in years.

- Overall data were categorized under SE and EH extremity. The categorization excludes the data of planetary landers, sample return and atmospheric probes, impactors, and rovers.

- Statistical analysis of non-parametric (Kaplan-Meier estimation) and parametric (Weibull probability) estimation were performed over the spacecraft's data in terms of iteration-1 (for SE extremity) and iteration2 (for EH Extremity).

- Then, we perform the same procedure of reliability analysis over the spacecraft data of various mass category [Small (0-500kg) - Medium (500-2500 kg) - Large (>2500kg)] to compare and assert the reliability effects found by G.F.Dubos.

- Finally, with reference to the results obtained, we discuss the possible causes accountable for variance in reliability behaviour of spacecraft operating in the different interplanetary environment.

\section{B. Data Description and Categorization}

For our analysis, we gathered spacecraft data from [7-9] in terms of spacecraft's gross mass in kg and lifespan in years from the time frame of 1960 to 2020. The sample template of data collection is shown in table-1. And our database has a total of 131 spacecraft of successful, partial success, failed, and active missions. To ensure homogeneity, sister spacecraft with a similar lifespan is considered as one. Similarly, en-route space missions and eliminated from our database for further analysis (i.e., Emirates Mars Mission and Mars 2020 Rover). Finally, overall data were categorized under extremity conditions (i.e. out of 131 spacecraft, 82 spacecraft falls under SE-Extremity, and 49 under EH-Extremity).

Table 1 Sample Template of Data Collection

\begin{tabular}{lllll}
\hline \hline Spacecraft Name & Launch Date & Failure/Decay Date & Launch Mass (kg) & Lifespan (Years) \\
\hline Pioneer 5 & 11 Mar 1960 & 26 Jun 1960 & 63 & 0.30 \\
\hline Pioneer 6A & 16 Dec 1965 & 08 Dec 2000 & 63 & 34.94 \\
\hline$\ldots . .$. & $\ldots$ & $\ldots$ & $\ldots$. & $\ldots$. \\
\hline New Horizons & 19 Jan 2006 & XX Oct 2020* & 401 & 14.66 \\
\hline *lifespan estimated for ongoing missions as of October 2020
\end{tabular}

\section{Formulations}

\section{A. Kaplan-Meier Estimation}

Kaplan-Meier is a non-parametric statistical estimation used to determine the survival function from the lifetime measurement of data. Here, we modify the equation to estimate the spacecraft reliability at different interplanetary boundaries based on their lifespan data. From the equation of Kaplan-Meier estimation [10], the modified expression for the reliability function of the spacecraft can be defined as 


$$
\widehat{\mathrm{R}}(\mathrm{t})=\prod_{\mathrm{t}_{\mathrm{i}} \leq \mathrm{t}}\left(1-\frac{\mathrm{s}_{\mathrm{i}}}{\mathrm{n}_{\mathrm{i}}}\right)
$$

$s_{i}$ - number of spacecraft operating or accomplished its mission intent

$n_{i}$ - number of spacecraft failed at time $\mathrm{t}$

$t_{i}$ - initial time after leaving low earth orbit

$t$ - time elapsed for the spacecraft to accomplish its intent/ time elapsed at the current operating state.

\section{B. Weibull Distribution}

Similar to Kaplan-Meier estimation, Weibull probability distribution (parametric function) is used to estimate the reliability analysis for electronic components and spacecraft subsystems. Here, we define the Weibull distribution function [11] for the spacecraft at appropriate extremity as

$$
\mathrm{S}_{\mathrm{f}}(\mathrm{t})=\frac{\beta}{\eta}\left(\frac{\mathrm{t}}{\eta}\right)^{\beta-1} \mathrm{e}^{-\left(\frac{\mathrm{t}}{\eta}\right)^{\beta}}
$$

where $\beta$ is the shape parameter, $\eta$ is the scale parameter, and $t$ is the lifespan of spacecraft in years.

Relation of Failure rate with $\beta$ : (i) $\beta<1$ defines that the failure rate decreases with time (lifespan), (ii) for $\beta \simeq 1$ the spacecraft has a fairly constant failure rate, and (iii) for $\beta>1$ the failure rate of the spacecraft increases with time(lifespan).

\section{Reliability of the Spacecraft}

Weibull Reliability expression for the spacecraft from parametric estimation can be defined as

$$
R(t)=e^{-\left(\frac{t}{n}\right)^{\beta}}
$$

where the reliability is the exponential function of scale parameter $\eta$ and shape parameter $\beta$.

\section{Hazard Function of the Spacecraft}

The hazard function or hazard rate $\lambda(t)$ of the spacecraft can be defined as the ratio of Weibull distribution function to Weibull reliability that can be mathematically written as

Mean Reliability and Median life of the Spacecraft

$$
\lambda(t)=\frac{\beta}{\eta}\left(\frac{t}{\eta}\right)^{\beta-1}
$$

The expression for the mean and median life of the spacecraft can be defined from the Weibull distribution function that can be written as

$$
\text { Mean Reliability } \overline{\mathrm{R}}=\eta \Gamma\left(\frac{1}{\beta}+1\right)
$$

where $\Gamma$ is the gamma function $\Gamma(\mathrm{n})=\int_{0}^{\infty} \mathrm{e}^{-\mathrm{t}} \mathrm{t}^{\mathrm{n}-1} \mathrm{dt}$

$$
\text { Median Life } \quad \tau=\eta(\ln (2))^{\frac{1}{\beta}}
$$

Variance from Weibull Distribution Function

$$
\text { Variance }=\eta^{2}\left[\Gamma\left(1+\frac{2}{\beta}\right)-\left(\Gamma\left(1+\frac{1}{\beta}\right)\right)^{2}\right]
$$

\section{V.Non-Parametric and Parametric Analysis of Spacecraft Reliability}

\section{A. Non-Parametric Kaplan-Meier Estimation}

Our database holds three types of data samples 1) time to the successful mission accomplishment, 2) time to the failure of spacecraft, and 3) time to the ongoing/active missions. This study intends to understand the reliability behaviour of the spacecraft when exposed to the distinct interplanetary environment. So, we have performed a powerful Kaplan-Meier estimation with random data censoring. The lifespan of the spacecraft in years was inputted as time range with random data censoring. 


\section{B. Parametric Weibull Probability Distribution}

To understand the analogy of reliability behaviour exhibited by interplanetary spacecraft by the Kaplan-Meier method, we perform the Weibull probability distribution function over the spacecraft data. The procedure follows random data censoring and Weibull analysis over the lifespan (in years) of spacecraft by maximum likelihood estimation of single Weibull fit. Relevant equations and formulations are shown in equation (1-8).

\section{VI.Results of Non-Parametric and Parametric Analysis}

A. Non-Parametric Results for SE-Extremity: Kaplan-Meier estimation shows that the spacecraft operating within the boundary of Sun and Earth exhibits $48 \%$ reliability after 2 years of functioning and can extend their performance to $\simeq 30 \%$ reliability after 6 years. At this extremity, the spacecraft experiences a hazard rate of $10 \%$ after 4 years and $20 \%$ after 15 years of operation. The reliability behavior of spacecraft at SE-Extremity is shown in Fig 1.

B. Non-Parametric Results for EH-Extremity: Comparably for the spacecraft operating between the bounds of Earth and the farthest point of the Solar System (say Heliopause) exhibits 60\% reliability after 2 years of operation and extends up to $40 \%$ reliability after 6 years. Spacecraft within this extremity is exposed to a hazard rate of $10 \%$ after 6 years and $20 \%$ after 18 years. It can be understood from Fig 2.
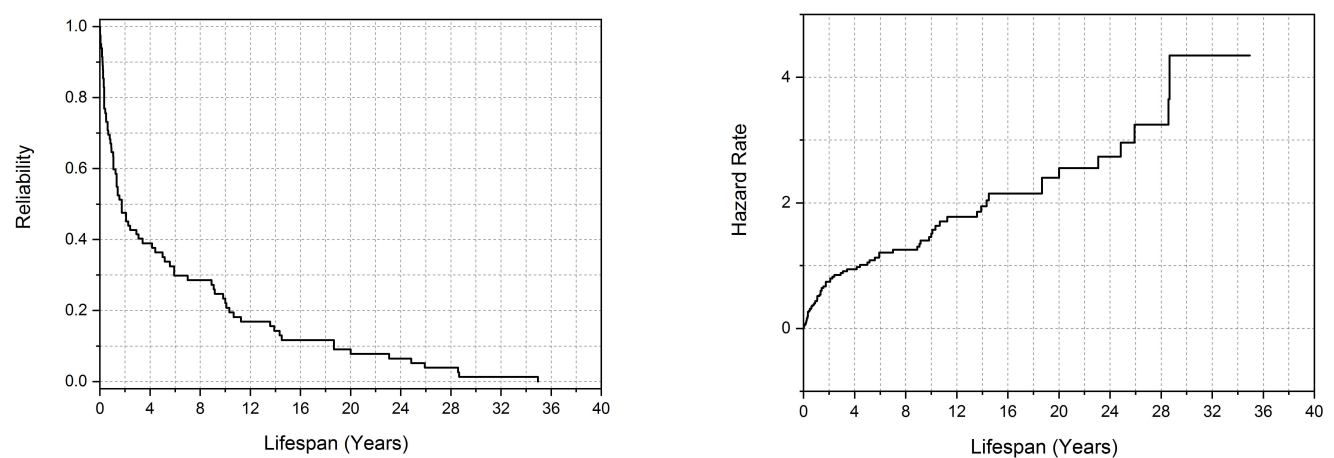

Fig 1 Kaplan-Meier Curve for Reliability and Hazard Rate of Interplanetary Spacecraft at SE-Extremity
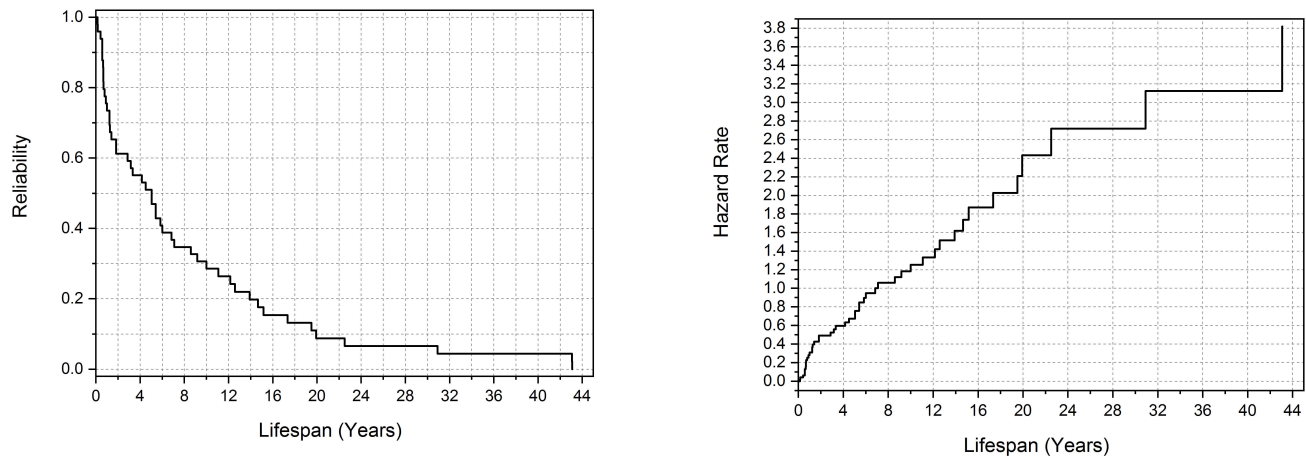

Fig 2 Kaplan-Meier Curve for Reliability and Hazard Rate of Interplanetary Spacecraft at EH-Extremity

C. Parametric Results for SE-Extremity: Parametric estimation using Weibull Distribution showed that the spacecraft operating within the boundary of SE-Extremity reflects 35\% reliability after 4 years of operation with $95 \%$ upper confidence of $48 \%$ and lower confidence of $\mathbf{3 0 \%}$. The spacecraft can function up to a maximum extent of 36 to 38 years. At this extremity, the spacecraft encounters a $20 \%$ hazard rate after 2.5 years that gradually decreases with an increase in lifespan of space probes shown in Fig 3.

D. Parametric Results for EH-Extremity: Contrary to the SE-Extremity, the spacecraft functioning between the boundary of Earth and to a limited extent of Solar System shows 52\% reliability after 4 years of operation with $95 \%$ upper confidence of $70 \%$ and lower confidence of $48 \%$. The spacecraft at this extremity experiences a $10 \%$ hazard rate after 12.5 years of operation which readily decreases relative to an increase in lifespan. This effect can be realized from Fig 4. Further, the spacecraft at this boundary can last up to an upper limit of 48 years of operation. 

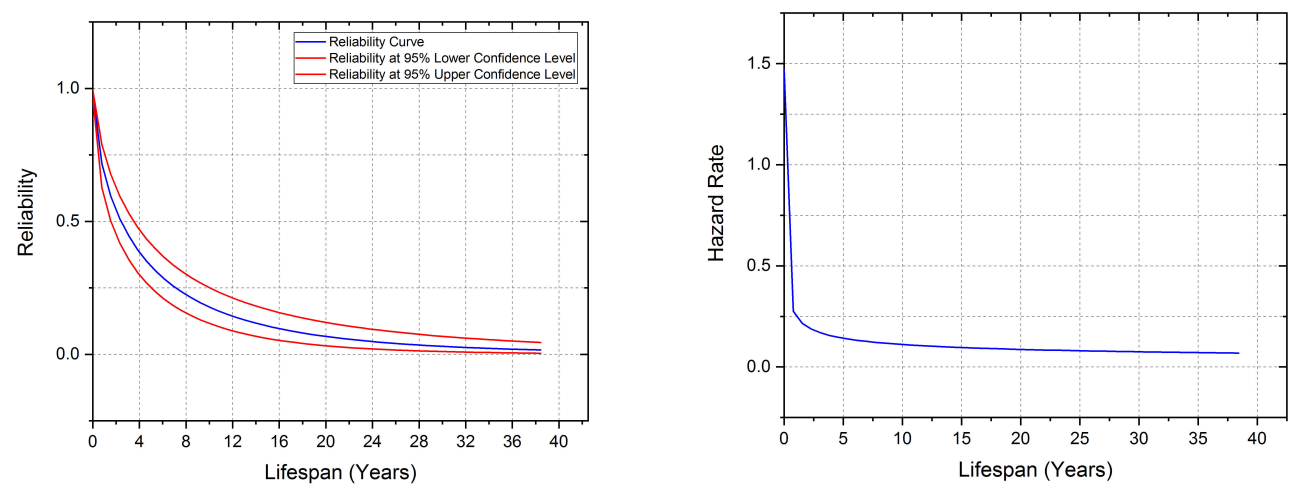

Fig 3 Weibull Curve for Reliability and Hazard Rate of Interplanetary Spacecraft at SE-Extremity
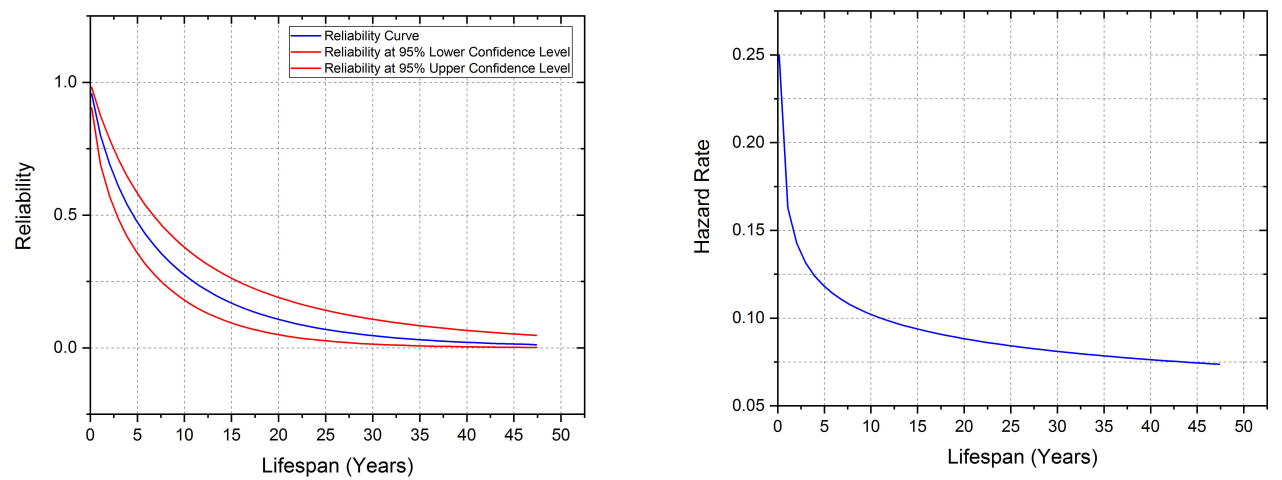

Fig 4 Weibull Curve for Reliability and Hazard Rate of Interplanetary Spacecraft at EH-Extremity

\section{E. Comparison of Results}
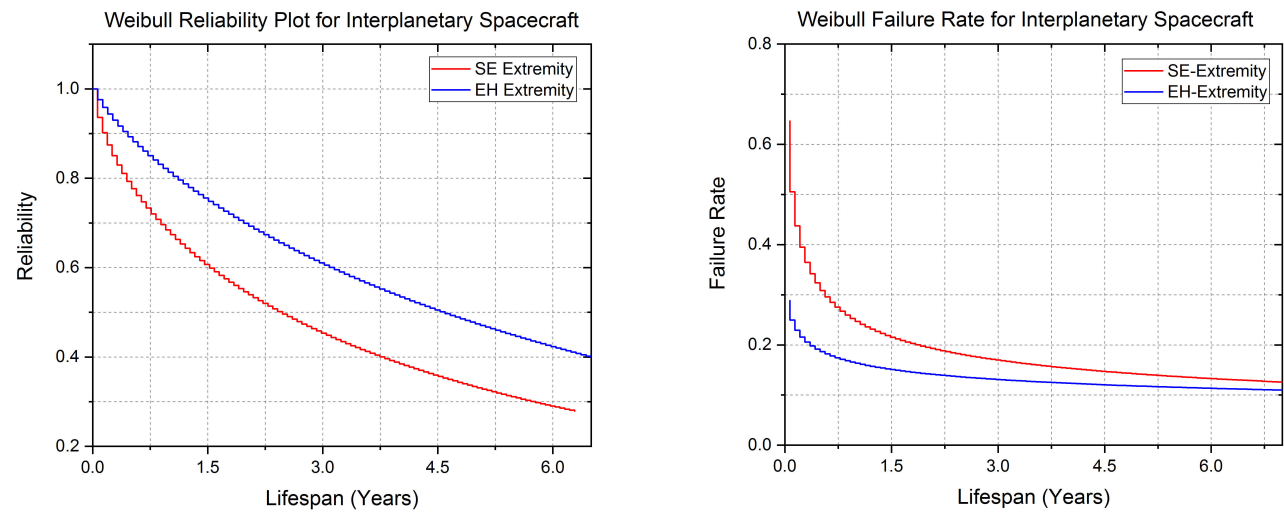

Fig 5 Weibull Reliability and Failure Rate Plot for Interplanetary Spacecraft at SE \& EH-Extremity

Comparing both parametric and non-parametric results in terms of reliability behaviour and hazard rate of interplanetary spacecraft inhered in two interplanetary boundaries, we have found that the boundary limit from the Earth to the Heliopause renders a promising environment for substantial reliability than the Sun-Earth boundary. Our analysis shows that the approximate mean reliability of spacecraft at SE-Extremity is 58\% which is smaller than $74 \%$ at EH-Extremity with a variance of $\operatorname{Var}_{S E}=0.0238$ to $\operatorname{Var}_{E H}=0.0145$ shown in table 4. More precisely the reliability behaviour can be understood from the equations

$$
\mathrm{R}(\mathrm{t})_{\mathrm{SE}}=\mathrm{e}^{-\left(\frac{\mathrm{t}}{4.29271}\right)^{0.64439}} \text { and } \mathrm{R}(\mathrm{t})_{\mathrm{EH}}=\mathrm{e}^{-\left(\frac{\mathrm{t}}{7.23227}\right)^{0.79025}} \quad \text { (9) and (10) }
$$


Similarly, both extremity shows a shape parameter ( $\beta=0.644$ at SE-Extremity) and ( $\beta=0.790$ at EH-Extremity) shown in table 4 . And overall shape parameter is found to be less than unity (i.e. $\beta<1$ ). The parameter $\beta<1$ depicts the failure rate or the hazard rate decreases with an increase in lifespan that can be precisely understood from the expressions,

$$
\lambda(\mathrm{t})_{\mathrm{SE}}=0.150\left(\frac{\mathrm{t}}{4.29271}\right)^{0.64439-1} \text { and } \lambda(\mathrm{t})_{\mathrm{SE}}=0.109\left(\frac{\mathrm{t}}{7.23227}\right)^{0.79205-1}
$$

Further, the results and estimates from the non-parametric and parametric analyses are shown in the table-2. Estimated values calculated from equation $(5,7,8)$ are shown in table 4.

Table 2 Mean and Quartile Estimate from Kaplan-Meier Analysis

\begin{tabular}{llllllllll}
\hline Quartile Estimate & \multicolumn{3}{l}{ SE-Extremity } & \multicolumn{3}{l}{ EH- Extremity } & Mean Estimate & SE-Extremity & EH-Extremity \\
\hline Percent Failures & 25 & 50 & 75 & 25 & 50 & 75 & Estimate & 5.9393 & 8.2871 \\
\hline Estimate & 0.49 & 1.74 & 9.16 & 1.00 & 5.05 & 12.16 & Standard Error & 0.9128 & 1.4950 \\
\hline 95\% LCL & 0.31 & 1.08 & 5.00 & 0.66 & 1.83 & 6.83 & $95 \%$ LCL & 4.1501 & 5.3568 \\
\hline 95\% UCL & 0.92 & 3.41 & 13.58 & 1.83 & 7.08 & 17.33 & $95 \%$ UCL & 7.7284 & 11.2174 \\
\hline \hline
\end{tabular}

Table 3 Estimate from Weibull Probability Distribution Function

\begin{tabular}{lllllllll}
\hline \hline Weibull Parameters & \multicolumn{2}{l}{ SE-Extremity } & \multicolumn{5}{l}{ EH-Extremity } \\
\hline DF & 1 & 1 & 1 & 1 & 1 & 1 & 1 & 1 \\
\hline Estimate & 1.4569 & 1.5518 & 4.2927 & 0.6443 & 1.9785 & 1.2654 & 7.2322 & 0.7902 \\
\hline Standard Error & 0.1820 & 0.1356 & 0.7816 & 0.0563 & 0.1918 & 0.1422 & 1.3875 & 0.0888 \\
\hline 95\% LCL & 1.1000 & 1.3075 & 3.0043 & 0.5429 & 1.6025 & 0.0152 & 4.9656 & 0.6340 \\
\hline $\mathbf{9 5 \%}$ UCL & 1.8137 & 1.8417 & 0.5429 & 0.7647 & 2.3545 & 1.5772 & 10.5336 & 0.9849 \\
\hline \hline
\end{tabular}

Table 4 Parametric Results of Spacecraft Reliability

\begin{tabular}{llll}
\hline \hline Results & Notation & SE-Extremity & EH-Extremity \\
\hline Shape Parameter & $\beta$ & 0.64439 & 0.79025 \\
\hline Scale Parameter & $\eta$ & 4.29271 & 7.23227 \\
\hline Variance & Var & 0.0238 & 0.0145 \\
\hline Mean Reliability & $\bar{R}$ & 0.586 & 0.740 \\
\hline Mean Life & $\tau$ & 2.4316 & 4.5413 \\
\hline Standard Deviation & $\sigma$ & 0.154330 & 0.120698 \\
\hline \hline
\end{tabular}

\section{VII.Hypothesis on Possible Causes responsible for distinct Reliability behaviour}

\section{A. Distribution of Cosmic Radiation over Interplanetary Space}

High energetic particles from Cosmic rays and Galactic cosmic rays greatly affect and cause rupture to the electronic components of the spacecraft. Notable incidence is the damage of electronic chips aboard the spacecraft computer of the Fobos-Grunt mission [12]. Galactic cosmic rays, solar cosmic rays, and solar particle events are the natural phenomena of the space environment. High energetic particles from these phenomena can cause damage to the on-board circuitry system of spacecraft thereby rupturing the electronic components and hardware. Notable incidences were the damage of the electrical system of Nozomi and Phobos spacecraft on their interplanetary transit from Earth to Mars [12]. So, the spacecraft operating within the bounds of SE-Extremity have increased vulnerability to the solar particle event and solar flares or eruption that ultimately impairs the circuitry system. Since the SEExtremity lies in close proximity to the Sun with a distance of $0.4-1.0$ AU. Comparably the spacecraft functioning within the bounds of EH-Extremity exhibits substantial reliability because the spacecraft has decreased vulnerability to the harmful effects of solar events as well as the intensity of cosmic rays. Observation from the radiation measurement of Pioneer and Voyager spacecraft showed that the intensity of cosmic radiation gradually decreases as we move far from the SE-Extremity [13-16]. Since solar events play a significant role in the distribution of radiation throughout the solar system environment [17-18]. Hence we conclude that the EH-Extremity is found to have a promising environment for the interplanetary spacecraft to operate with extended reliability.

\section{B. Power Source of the Spacecraft}

Spacecraft power plays a principal component in spacecraft's reliability. Major interplanetary spacecraft encounters a challenge in power generation at both extremities. Because the solar panels of the spacecraft operating at SE-Extremity get depleted with time due to the extreme temperature and collision of hazardous particles during 
solar eruptions or events, resulting in the loss of power and spacecraft function. Therefore, spacecraft malfunction proportionate decreased reliability. In parallel to the mission operation, solar power generation poses a challenging task for the spacecraft operating at EH-Extremity as the intensity of solar irradiance decreases from SE to the EH Extremity and remain unavailable during solar conjunctions [21]. But the potential cause for the increased reliability behaviour at EH-Extremity may be due to the power source, i.e. the magnificent and extended operating probes like Voyager 1 and 2, and New Horizons that embraces nuclear thermoelectric generators as their primary power source which provides uninterrupted power supply throughout the mission life and enhancing reliability [22-24].

\section{Space Environment and Temperature}

The region of interplanetary space between the Sun and the Heliopause is habituated with a hostile space environment adversely affecting the spacecraft's operation and reliability. The region at SE-Extremity experiences extreme temperature that ultimately affects the stabilization of the spacecraft's internal temperature. Further, the probe's internal temperature assists the spacecraft in the management of on-board fuel storage plighted for attitude control and course-correcting maneuver and determines the efficiency or lifetime of electronic components. Hence this hostile environment at SE-Extremity prompts a potential impact on spacecraft reliability whereas the environment at EH-Extremity experiences curtailed temperature due to the non-availability of adequate solar irradiance [21]. Thus the EH-Extremity affords an auspicious environment for nuclear-powered spacecraft to maintain the probe's internal thermal stability and improved reliability as compared to the SE-Extremity.

\section{Impact of Spacecraft Mass}

The gross mass of the spacecraft (which includes the spacecraft's dry mass + fuel mass) has a significant contribution to their reliability. But here the fuel mass has a direct role in the determination of spacecraft reliability. The spacecraft is fuelled to perform maneuver correction and to stabilize the attitude control system to remain itself in a stabilized orbit around the planetary body. So, fuel depletion results in failure of orientation and attitude control system resulting in the disposal of spacecraft despite the perfect functioning of spacecraft's sub-system. Notable spacecraft encountered this issue is ESA's Cassini Spacecraft that finally plunged into Saturn's atmosphere [25]. Considerably both extremities possess a challenging environment for fuel management and cryogenic storage to maintain zero boil-off, the SE-Extremity may endorse fuel depletion and the EH-Extremity may spawn fuel solidification (Nozomi's fuel solidification during interplanetary transit [12]). These circumstances may result in a malfunction of attitude thrusters and a drop in reliability. Hence, the spacecraft with a considerable amount of fuel mass within a mass limit of $500-2500 \mathrm{~kg}$ is desirable for a reliable mission.

\section{E. Spacecraft Components, Design, Testing, and Integration}

The quality of electronic components and hardware, dimension of the spacecraft, its attentive integration and testing affects the reliability of the spacecraft. For example, the standard of electronic components manufactured during the 1970 s that are integrated with the series of interplanetary spacecraft's like Pioneer $10 \& 11$ (that operated for more than 20 years), Voyager $1 \& 2$, and New Horizons in 2006 are still functioning and has crossed their estimated mission lifetime [26-27]. But modern electronic components have reduced dimension with uncertainty in their reliability. Further, large spacecraft is associated with complex hardware and wiring configuration that has increased the chances of human error during the integration of spacecraft in ground-based laboratories. Furthermore, lack of adequate shielding and design, testing and validation of spacecraft's sub-system, and negligent fabrication are the other factors substantially influencing the spacecraft's reliability.

\section{VIII.Testification of Reliability Effect Interpreted by G.F.Dubos.2010}

\section{A. Data Categorization and Censoring}

In this section, the spacecraft data were sorted in ascending order concerning the gross mass. Then the overall data were distributed to the mass column of distinct mass category shown in table 5.

Table 5 Spacecraft Categorization

\begin{tabular}{llll}
\hline \hline Spacecraft's Mass Category & Mass Range & Example at SE-Extremity & Example at EH-Extremity \\
\hline Small & $0-500 \mathrm{~kg}$ & Helios A \& B, Genesis & Mariner 4, New Horizons \\
\hline Medium & $500-2500 \mathrm{~kg}$ & Venera 11 \& 12, Messenger & Solar Orbiter, Voyager 1 \& 2 \\
\hline Large & $>2500 \mathrm{~kg}$ & Venera 14 and 15 & Tianwen-1 \\
\hline \hline
\end{tabular}


There were 50 spacecraft in the small category, 55 spacecraft in the medium-sized category, and 26 spacecraft in the large category. These sorted data were analyzed over powerful Kaplan-Meier estimation and then with Weibull probability fit with random data censoring. Our analysis executed here is to address the question of whether the spacecraft's mass affects reliability. And to assert the reliability effect interpreted by Duboset.al.2010 employing Castet and Saleh spacecraft's reliability model $[1,6]$.

\section{IX.Non-Parametric and Parametric Results for Mass Category}

\section{A. Non-Parametric Results (Kaplan-Meier)}

Small Category: Our analysis showed that the spacecraft exhibits $\simeq 50 \%$ reliability after 2.5 years of operation in interplanetary space, and extends up to 10 years with $20 \%$ reliability. Spacecraft of this category can function up to a maximum lifespan of 20-25 years.

Medium Category: The spacecraft under this category exhibited excellent reliability than the other two mass categories. Our analysis showed that the spacecraft operates with $60 \%$ reliability after 2.5 years of continuous operation and extends up to 5 years with 55\% reliability. It continues its operation with the efficiency of $20 \%$ reliability for 13 years in interplanetary space. The maximum estimated lifespan of the spacecraft of this category is 25-30 years.

Large Category: This category spacecraft exhibits $\simeq 30 \%$ reliability after 2.5 years of operation with a hazard rate of $15 \%$ and the reliability readily drops to $10 \%$ after 7 years with a hazard rate of $25 \%$ at 7.5 years. Space probes having this mass limit is expected to function up to a maximum lifespan of 10-13 years. The reliability and Hazard rate estimated from the parametric analysis is shown in Fig 6.
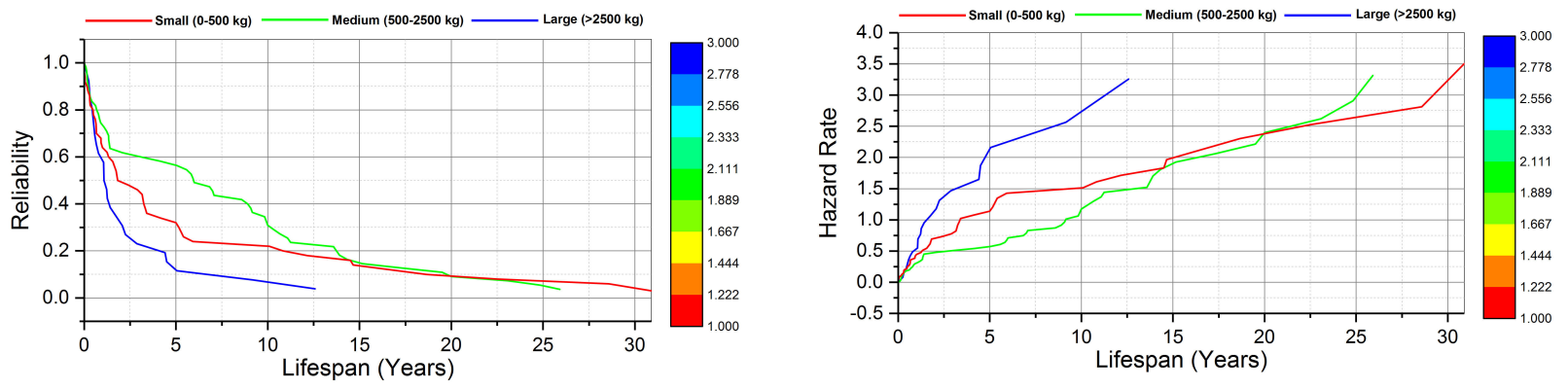

Fig 6 Kaplan-Meier Estimation: Reliability and Hazard Rate Plot for Various Mass Category

\section{B. Parametric Results (Weibull Fit)}

Small Category: Weibull analysis showed that the spacecraft exhibits $\simeq 30 \%$ reliability after 7 years of operation with $95 \%$ upper confidence of $40 \%$ reliability and lower confidence of $20 \%$. The overall hazard rate of $1 \%$, the probe experiences during their period of operation from 10-20 years.

Medium Category: Medium-sized spacecraft exhibits $\simeq 48 \%$ reliability after 5 years of functioning with $95 \%$ upper confidence of $60 \%$ reliability and lower confidence of $30 \%$. The spacecraft of this category is found to have an overall hazard rate of $1 \%$ after 10 years of operation that gradually decreases relative to an increase in lifespan.

Large Category: Large-sized spacecraft having mass $>2500 \mathrm{~kg}$ exhibits $\simeq 20 \%$ reliability after 5 years of operation with $95 \%$ upper confidence of $50 \%$ and lower confidence of $10 \%$ reliability. Spacecraft having this much mass limit is found to have a maximum hazard rate of $3 \%$ after 5 years of operation and a minimum of $2 \%$ after 10 years. The reliability behaviour of spacecraft of various mass category and its hazard rate is shown in Fig 7-8.

Weibull Probability for Spacecraft Reliability

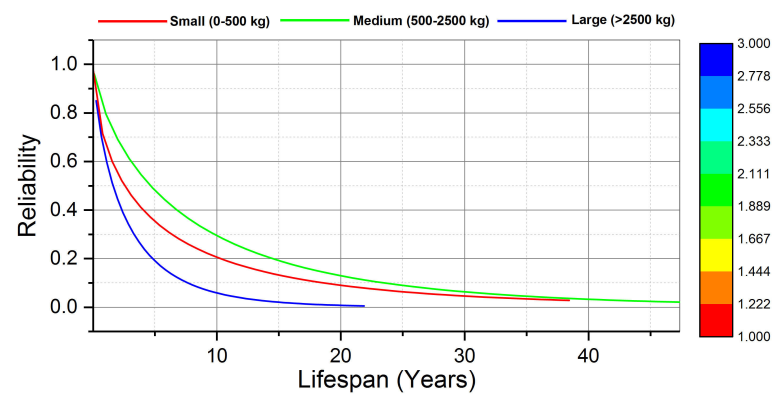

Weibull Probability for 95\% Lower Confidence Level

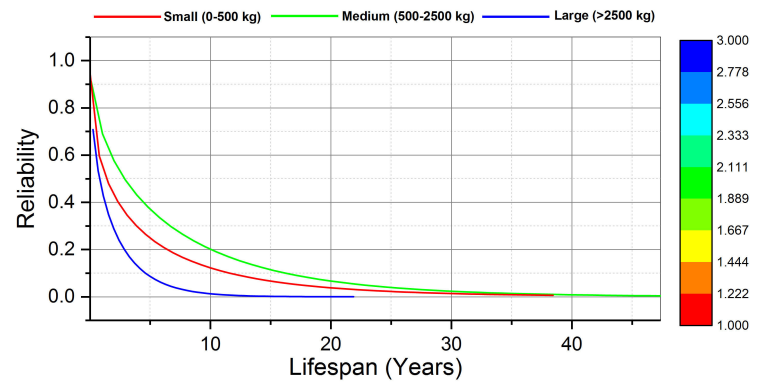

Fig 7 Weibull Analysis: Reliability and 95\% LCL Reliability Plot for Various Mass Category 

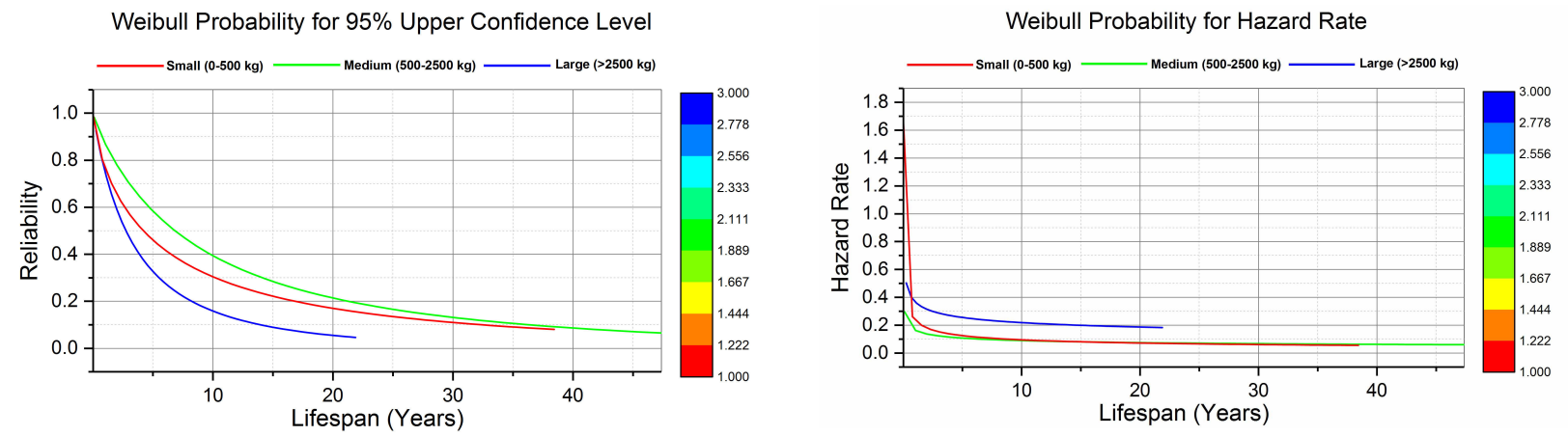

Fig 8 Weibull Analysis: 95\% UCL Reliability and Hazard Rate Plot for Various Mass Category

\section{Comparison of Results}

In contrast to the results, medium-sized spacecraft exhibits superior reliability than the other two mass categories with the least hazard rate of $1 \%$. It can be realized from the expression below

$$
\begin{gathered}
\text { Reliability, } \mathrm{R}(\mathrm{t})_{\text {Medium }}=\mathrm{e}^{-\left(\frac{\mathrm{t}}{7.63407}\right)^{0.74117}} \\
\text { Hazard Rate, } \lambda(\mathrm{t})_{\text {Medium }}=0.097\left(\frac{\mathrm{t}}{7.63407}\right)^{0.74118-1}
\end{gathered}
$$

where $\lambda(t)_{\text {Medium }}$ is the hazard function that decreases with an increase in the lifespan of medium-sized spacecraft.

The resultant plot for the Reliability and Hazard rate of the spacecraft from scale and shape parameter of various mass categories is shown in Fig 9. Further, Mean and Quartile estimate from Kaplan-Meier estimation and Weibull probability distribution is shown in table 6-8.
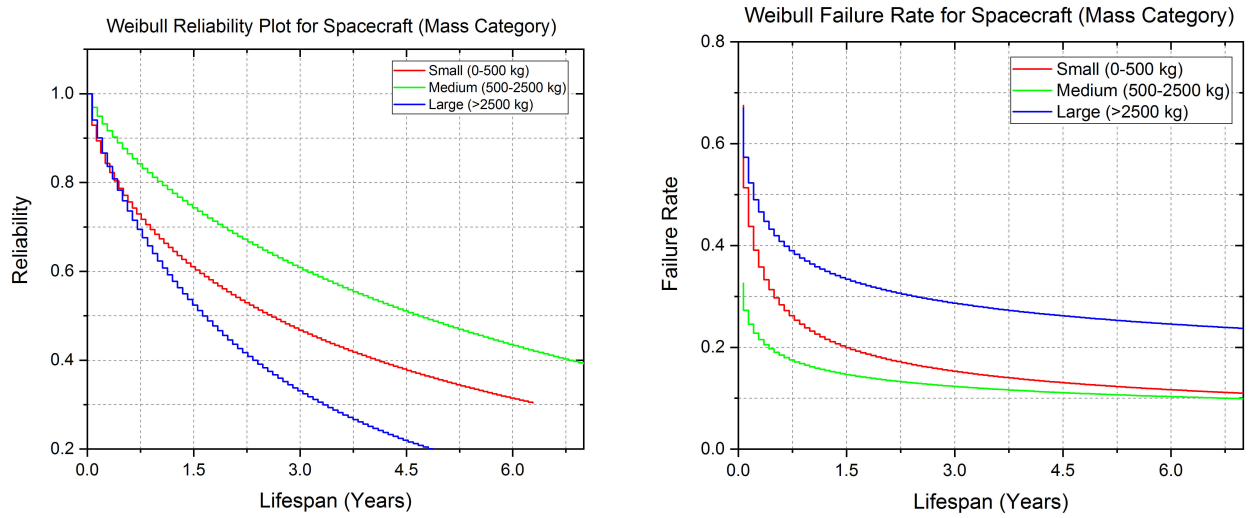

Fig 9 Weibull Probability: Reliability and Hazard Rate Plot for Various Mass Category

Table 6 Mean Estimate from Kaplan-Meier Analysis

\begin{tabular}{llll}
\hline \hline Mean Estimate & Small Category & Medium Category & Large Category \\
\hline Estimate & 6.4262 & 8.5254 & 2.9407 \\
\hline Standard Error & 1.3071 & 1.2933 & 0.8692 \\
\hline 95\% LCL & 3.8642 & 5.9905 & 1.2371 \\
\hline 95\% UCL & 8.9881 & 11.0603 & 4.6444 \\
\hline \hline
\end{tabular}

Table 7 Quartile Estimate from Kaplan-Meier Analysis

\begin{tabular}{llllllllll}
\hline \hline Quartile Estimate & \multicolumn{3}{l}{ Small Category } & \multicolumn{3}{c}{ Medium Category } & \multicolumn{3}{c}{ Large Category } \\
\hline Percent Failures & 25 & 50 & 75 & 25 & 50 & 75 & 25 & 50 & 75 \\
\hline Estimate & 0.65 & 1.83 & 5.93 & 0.9 & 6 & 11.25 & 0.58 & 1.08 & 2.87 \\
\hline 95\% LCL & 0.31 & 1.25 & 3.33 & 0.42 & 2.08 & 9.16 & 0.33 & 0.66 & 1.26 \\
\hline 95\% UCL & 1.33 & 3.41 & 14.66 & 2.08 & 9.16 & 15.16 & 1.08 & 2.08 & 5.05 \\
\hline \hline
\end{tabular}


Table 8 Estimate from Weibull Probability Distribution

\begin{tabular}{|c|c|c|c|c|c|c|c|c|c|c|c|c|}
\hline \multirow{2}{*}{$\begin{array}{l}\text { Parameters } \\
\text { DF }\end{array}$} & \multicolumn{4}{|c|}{ Small Category } & \multicolumn{4}{|c|}{ Medium Category } & \multicolumn{4}{|c|}{ Large Category } \\
\hline & 1 & 1 & 1 & 1 & 1 & 1 & 1 & 1 & 1 & 1 & 1 & 1 \\
\hline Estimate & 1.5467 & 1.6517 & 4.6960 & 0.6054 & 2.0326 & 1.3492 & 7.6340 & 0.7411 & 0.9586 & 1.2916 & 2.6082 & 0.7742 \\
\hline Standard Error & 0.2478 & 0.1885 & 1.1637 & 0.0691 & 0.1924 & 0.1511 & 1.4693 & 0.0830 & 0.2704 & 0.1887 & 0.7054 & 0.1131 \\
\hline $95 \%$ LCL & 1.0610 & 1.3206 & 2.8893 & 7.6326 & 1.6553 & 1.0831 & 5.2351 & 0.5950 & 0.4285 & 0.9700 & 1.5350 & 0.5814 \\
\hline $95 \%$ UCL & 2.0324 & 2.0658 & 7.6326 & 0.7572 & 2.4098 & 1.6805 & 11.1322 & 0.9232 & 1.4887 & 1.7198 & 4.4315 & 1.0309 \\
\hline
\end{tabular}

Table 9 Parametric Results of Spacecraft Reliability (Mass Category)

\begin{tabular}{lllll}
\hline \hline Results & Notation & Small Category & Medium Category & Large Category \\
\hline Shape Parameter & $\beta$ & 0.6054 & 0.7411 & 0.7742 \\
\hline Scale Parameter & $\eta$ & 4.6960 & 7.6340 & 2.6082 \\
\hline Variance & Var & 0.0180 & 0.0116 & 0.0802 \\
\hline Mean Reliability & $\bar{R}$ & 0.5538 & 0.6964 & 0.6877 \\
\hline Mean Life & $\tau$ & 2.5614 & 4.6518 & 1.6216 \\
\hline Standard Deviation & $\sigma$ & 0.1343 & 0.1079 & 0.2833 \\
\hline \hline
\end{tabular}

\section{Possible causes accountable for Reliability behaviour of Spacecraft of Various Mass Category}

We have not discussed much the possible causes behind the reliability behaviour of spacecraft of distinct mass category, because the significance and possible causes were clearly explained in [6]. However, the role of spacecraft reliability in terms of mass category in interplanetary space was discussed in section 7 "Impact of Spacecraft Mass".

\section{Conclusion}

Reliability of the spacecraft is one of the significant parameters to ascertain the extent of mission proficiency and competency. Numerous space environmental factors enhance and recede reliability. To realize whether the spacecraft at distinct interplanetary extremity exhibits different reliability, we performed statistical analysis employing KaplanMeier and Weibull Probability distribution over spacecraft data. Our analysis concluded that the expanse of the interplanetary boundary between the Earth and the farthest point of the solar system renders a protecting and favouring environment for sustainable and reliable missions. In addition to this, reliability analysis of interplanetary spacecraft of the different mass categories using the Castet-Saleh model showed that spacecraft of a medium category (with a mass limit of 500-2500 kg) appears to reflect competent reliability than small and large category. Further, we have discussed the potential causes responsible for the difference in the reliability behaviour of spacecraft operating at the distinct interplanetary boundary. Finally, we expect that our work may provide a useful framework for space agencies and spacecraft manufacturers to contemplate adequate spacecraft design and integration along with the perspective of interplanetary boundary selection for future interplanetary missions.

\section{Acknowledgements}

I would like to extend my sincere thanks to the General Manager Mr. Raajesh Ghoyal, Pulkit Metals Private Limited, Pondicherry, India for the financial aid for the research. Further, I would like to acknowledge my research guide Dr. A. Ramesh Naidu. Ph.D, for his kind and wholehearted support throughout this research work.

\section{Dedication}

I would like to dedicate my research work to my beloved mother late. Mrs. Malathi Biswal for her motivational speech and emotional support throughout my life.

\section{References}

[1] Castet, J. F., \& Saleh, J. H. (2009). Satellite reliability: statistical data analysis and modeling. Journal of Spacecraft and Rockets, 46(5), 1065-1076.

[2] Castet, J. F., \& Saleh, J. H. (2009). Satellite and satellite subsystems reliability: Statistical data analysis and modeling. Reliability Engineering \& System Safety, 94(11), 1718-1728.

[3] Tafazoli, M. (2009). A study of on-orbit spacecraft failures. Acta Astronautica, 64(2-3), 195-205.

[4] Biswal M, M. K., \& Naidu Annavarapu, R. (2020). Assessment of Efficiency, Impact Factor, Impact of Probe Mass, Probe Life Expectancy, and Reliability of Mars Missions. arXiv e-prints, arXiv:2009.08534.

[5] Biswal M, M. K., \& Naidu Annavarapu, R. (2021). A Study on Mars Probe Failures. In AIAA Science and Technology 2021 Forum and Exposition. (Submitted)

[6] Dubos, G. F., Castet, J. F., \& Saleh, J. H. (2010). Statistical reliability analysis of satellites by mass category: Does spacecraft size matter?. Acta Astronautica, 67(5-6), 584-595. 
[7] McDowell, J. (2001). Jonathan's Space Report - Satellite Catalog. Jonathan McDowell.

[8] Biswal M, Malaya Kumar; Annavarapu, Ramesh Naidu (2020), "Master Catalogue of Lunar and Mars Exploration Missions and their Probe Parameters", Mendeley Data, V2, doi: 10.17632/mdkzgz23dj.2

[9] Siddiqi, A. A., \& Launius, R. (2002). Deep space chronicle: A chronology of deep space and planetary probes 1958-2000.

[10] Kaplan, E. L., \& Meier, P. (1958). Nonparametric estimation from incomplete observations. Journal of the American statistical association, 53(282), 457-481.

[11] Rinne, H. (2008). The Weibull distribution: a handbook. CRC press.

[12] Biswal M, M. K., \& Naidu A, R. (2020). Mars Missions Failure Report Assortment: Review and Conspectus. In AIAA Propulsion and Energy 2020 Forum (p.3541). https://doi.org/10.2514/6.2020-3541.

[13] Stassinopoulos, E. G., \& Raymond, J. P. (1988). The space radiation environment for electronics. Proceedings of the IEEE, 76(11), 1423-1442.

[14] Fan, C. Y., Meyer, P., \& Simpson, J. A. (1960). Rapid reduction of cosmic-radiation intensity measured in interplanetary space. Physical Review Letters, 5(6), 269.

[15] Webber, W. R., \& Higbie, P. R. (2009). Galactic propagation of cosmic ray nuclei in a model with an increasing diffusion coefficient at low rigidities: A comparison of the new interstellar spectra with Voyager data in the outer heliosphere. Journal of Geophysical Research: Space Physics, 114(A2).

[16] Stone, E. C., Cummings, A. C., McDonald, F. B., Heikkila, B. C., Lal, N., \& Webber, W. R. (2013). Voyager 1 observes lowenergy galactic cosmic rays in a region depleted of heliospheric ions. Science, 341(6142), 150-153.

[17] Echer, E., Gonzalez, W. D., Guarnieri, F. L., Dal Lago, A., \& Vieira, L. E. A. (2005). Introduction to space weather. Advances in Space Research, 35(5), 855-865.

[18] Bourdarie, S., \& Xapsos, M. (2008). The near-earth space radiation environment. IEEE transactions on nuclear science, 55(4), 1810-1832.

[19] Mbarki, M., Sun, G. C., \& Bourgoin, J. C. (2004). Prediction of solar cell degradation in space from the electron-proton equivalence. Semiconductor science and technology, 19(9), 1081.

[20] Yamaguchi, M., Uemura, C., \& Yamamoto, A. (1984). Radiation damage in InP single crystals and solar cells. Journal of applied physics, 55(6), 1429-1436.

[21] Biswal M, M. K., \& Naidu A, R. (2021). Human Mars Exploration and Expedition Challenges. In AIAA Science and Technology 2021 Forum. (Submitted)

[22] de Winter, F., Stapfer, G., \& Medina, E. (1999). The Design of a Nuclear Power Supply with a 50 Year Life Expectancy: the JPL Voyager's SiGe MHW RTG (No. 1999-01-2586). SAE Technical Paper.

[23] Ottman, G., \& Hersman, C. (2006, June). The Pluto-new horizons RTG and power system early mission performance. In 4th International Energy Conversion Engineering Conference and Exhibit (IECEC) (p. 4029).

[24] O’Brien, R. C., Ambrosi, R. M., Bannister, N. P., Howe, S. D., \& Atkinson, H. V. (2008). Safe radioisotope thermoelectric generators and heat sources for space applications. Journal of Nuclear Materials, 377(3), 506-521.

[25] Edgington, S. G., \& Spilker, L. J. (2016). Cassini's grand finale. Nature Geoscience, 9(7), 472-473.

[26] Heacock, R. L. (1980). The Voyager Spacecraft. Proceedings of the Institution of Mechanical Engineers, 194(1), 211-224.

[27] Hall, C. F. (1983). The Pioneer 10/11 program: from 1969 to 1994. IEEE transactions on reliability, 32(5), 414-416.

\section{Appendices}
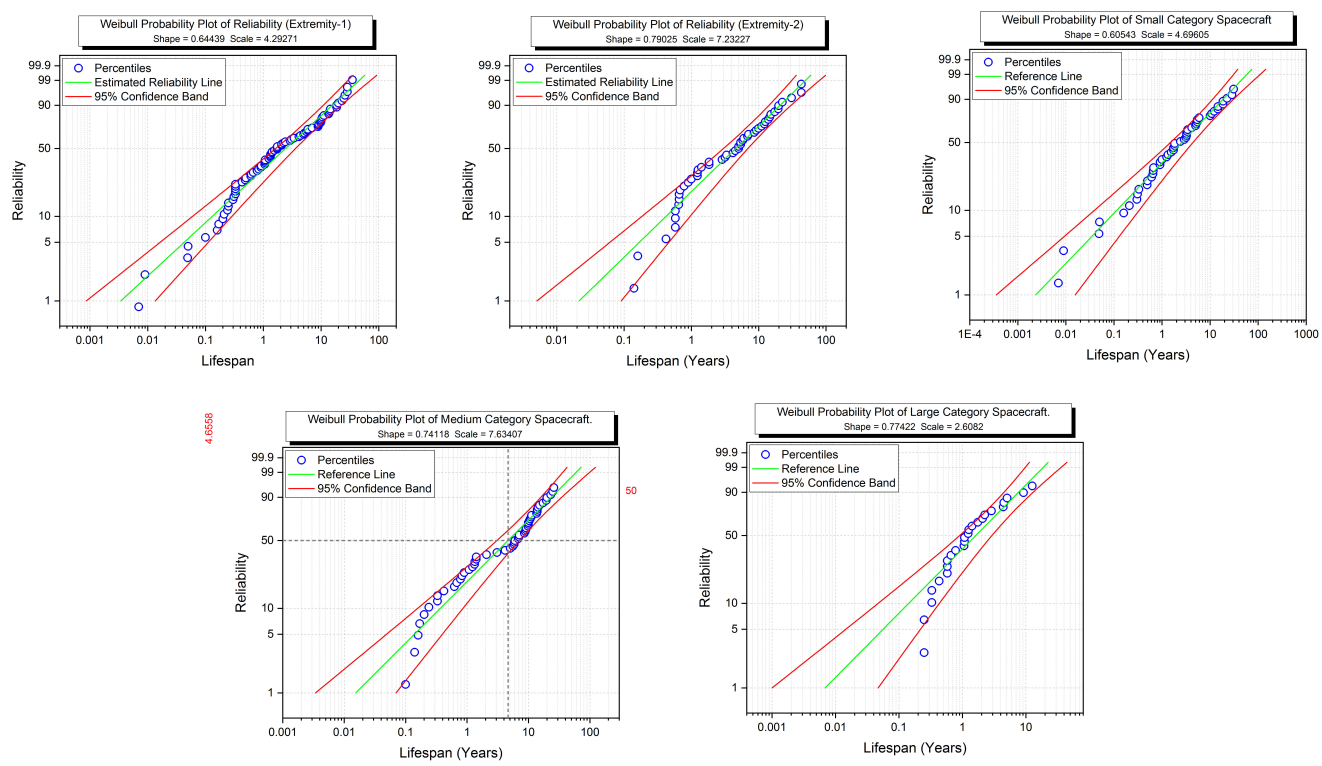

Weibull Probability Plot for SE \& EH- Extremity, and Small-Medium-Large Mass Category 